\title{
BMJ Open Efficacy of the bile leak test using contrast-enhanced intraoperative ultrasonic cholangiography in liver resection: a study protocol for a non- randomised, prospective, off-label, single-arm trial
}

Motofumi Tanaka, Masahiro Kido, Kaori Kuramitsu, Shohei Komatsu, Masahide Awazu, Hidetoshi Gon, Daisuke Tsugawa, Hideyo Mukubo, Hirochika Toyama, Takumi Fukumoto

To cite: Tanaka M, Kido M, Kuramitsu K, et al. Efficacy of the bile leak test using contrastenhanced intraoperative ultrasonic cholangiography in liver resection: a study protocol for a non-randomised, prospective, off-label, single-arm trial. BMJ Open 2019;9:e029330. doi:10.1136/ bmjopen-2019-029330

- Prepublication history for this paper is available online. To view these files, please visit the journal online (http://dx.doi. org/10.1136/bmjopen-2019029330).

Received 22 January 2019 Revised 23 April 2019 Accepted 21 May 2019
D) Check for updates

(c) Author(s) (or their employer(s)) 2019. Re-use permitted under CC BY-NC. No commercial re-use. See rights and permissions. Published by BMJ.

Hepato-Biliary-Pancreatic Surgery, Kobe University, Kobe, Japan

Correspondence to Dr Motofumi Tanaka motofutanaka-gi@umin.net

\section{ABSTRACT}

Introduction Bile leak is still a major complication after

liver resection to be improved. To intraoperatively detect this adverse complication, leak test is commonly performed after hepatic resection. However, by the conventional leak test, it is often difficult to know whether the test reagent reaches to intrahepatic bile duct near cut surface of liver with adequate volume and pressure to identify the existence of bile leak. Thus, in order to perform leak test more accurately, this study aims to evaluate the efficacy and safety of the leak test using contrast-enhanced intraoperative ultrasonic cholangiography (CE-IOUSC), which was reported by our group as a procedure for detection of bile duct.

Methods and analysis The current study is a nonrandomised, prospective, off-label, single-arm clinical trial for patients who undergo liver resection. A total of 100 patients will be enrolled. After completion of liver resection, the leak test is performed with CE-IOUSC using Sonazoid as a contrast agent to visualise dye injection into the intrahepatic bile duct. The primary endpoint is the success of the leak test, defined as clear visualisation of intrahepatic bile duct around cut surface by ultrasonography that indicates enough volume of dye injection. Secondary endpoints are postoperative bile leak and all adverse events related to CEIOUSC. The findings obtained through this study will establish this procedure to assist surgeons for adequately performing the leak test, precisely detecting intraoperative biliary leak strictly and reducing postoperative bile leak.

Ethics and dissemination The protocol is approved by Institutional Review Boards of Kobe University Hospital (No.290069). Our findings will be widely disseminated through conference presentations and peer-reviewed publications.

Trial registration number UMIN000031236 and jRCTs051180027.

\section{INTRODUCTION}

The postoperative mortality and morbidity after liver resection have markedly decreased

\section{Strengths and limitations of this study}

- Contrast-enhanced intraoperative ultrasonic cholangiography, which was originally developed by our institution, can visualise intrahepatic bile duct clearly enough and is expected to assist the leak test.

- To our knowledge, it may be the first study to evaluate utility of the biliary leak test, which is performed without any indicator of success.

- This study will help accurately perform the leak test, resulting in high detection rate of bile leak and reduction of postoperative bile leak.

- The single-arm and single-centre study design may limit general application of the purported results.

- Bile leakage from non-dependent site cannot be detected by this procedure, same as conventional test.

over the past few decades due to improvement in surgical technique and perioperative management. ${ }^{1-5}$ However, the incidence of bile leak has not parallelly decreased, ranging from $4.6 \%$ to $9.8 \%$ in recent studies. ${ }^{6-10}$ In many cases, although a posthepatectomy bile leak can be successfully managed by drainage of abdominal cavity, biliary stent using endoscopic retrograde cholangiography ${ }^{11}$ and antibiotics, it often results in longer hospital stay and, in some cases, can lead to organ space infection, sepsis and secondary liver failure.

To reduce incidence of the bile leak, intraoperative leak test after liver resection is commonly performed using air, saline or dye and has been reported to be effective $\mathrm{e}^{12-15}$ though evidence level is not high. ${ }^{16}$ Also, evaluation with the leak test is often difficult when occlusion of the distal common bile duct by 
hand or forceps for clamping is insufficient, for instance due to the anatomic variations in the level of cystic duct confluence. In such cases, whether an adequate volume of test reagent is injected into the intrahepatic bile duct cannot be judged even under visualisation by ultrasonography. ${ }^{914}$ In addition, inadequately high biliary pressure during injection can cause a secondary leak point, making it important to ensure suitable volume injection into the intrahepatic bile duct in the leak test.

We have previously reported the efficacy of contrast-enhanced intraoperative ultrasonic cholangiography (CE-IOUSC) in hepatobiliary surgery as a biliary navigation. ${ }^{17} 18$ It enables visualisation of the non-dilated intrahepatic bile duct by direct injection of perfluorobutane microbubbles (Sonazoid; Daiichi-Sankyo Pharmaceutical Co.) as a contrast agent of ultrasonography. ${ }^{17}$ Using this technique, it may be possible to know whether biliary injection of the reagent is adequate.

In this study, we have designed a study protocol for the leak test with CE-IOUSC after liver resection for evaluating the efficacy and safety of this procedure.

\section{METHODS AND ANALYSIS \\ Study design}

This study is a non-randomised, prospective, off-label, single-arm clinical trial. Approved by the institutional review committee, the study period is planned to be from 22 March 2018 to 31 March 2022. The registration period will end at 31 March 2021. Patients who visit the Kobe University Hospital during this period are the candidate participants. Patient condition will be followed up until the day of discharge from the hospital. The summary of the study is shown in figure 1 . The analysis period will continue until 1 year after the registration day. This study protocol follows SPIRIT-statement. ${ }^{19}$

\section{Study participants}

Potential participants are all patients who have an indication for liver resection during the study period, indicating patients with hepatic tumour, such as malignant and benign lesions, and living donors for liver transplantation.

\section{Inclusion criteria}

Participants meeting all the following criteria will be included:

1. Age at 20 years or older.

2. Indication for liver resection without biliary reconstruction.

3. Cholecystectomy to be performed at liver resection.

4. No history of side effect after venous injection of Sonazoid at preoperative examination.

5. Provision of written informed consent to participate in this study.

\section{Exclusion criteria}

Participants meeting any of the following criteria will be excluded:

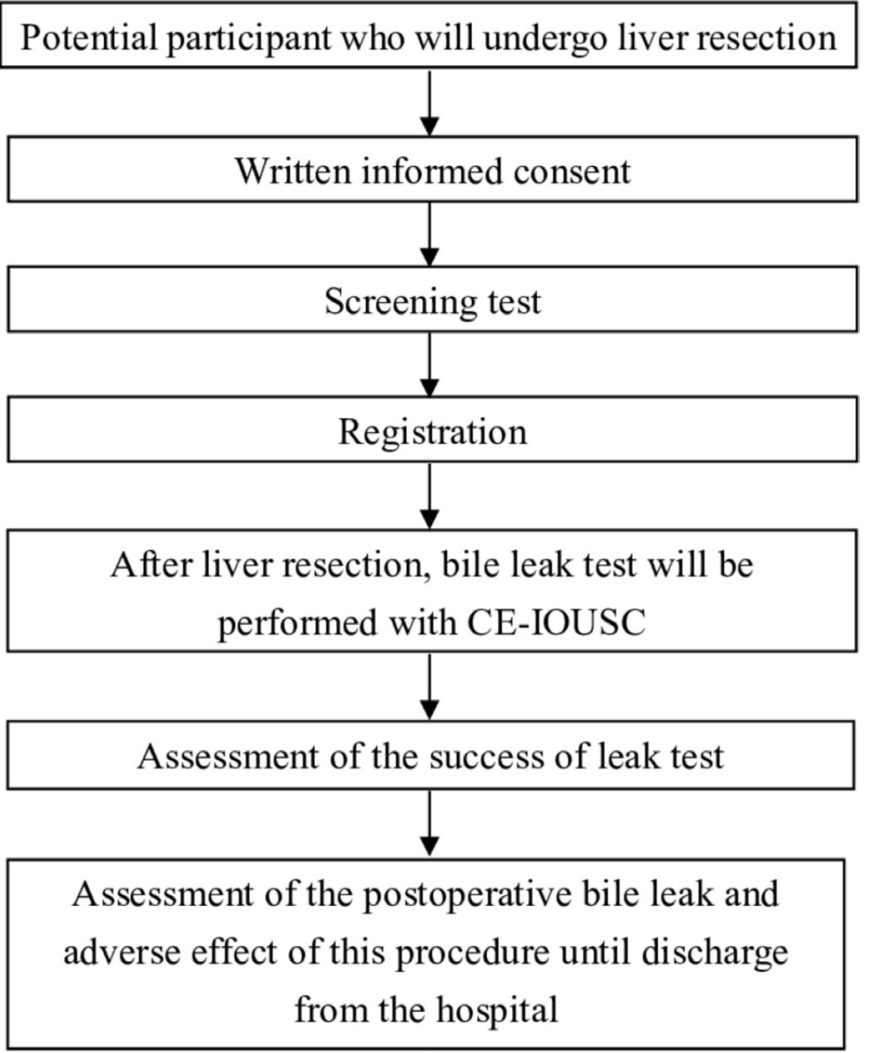

Figure 1 Summary of the study. CE-IOUSC, contrastenhanced intraoperative ultrasonic cholangiography.

1. Hypersensitivity for Sonazoid.

2. Allergic to egg or egg products.

3. Preoperative concomitant cholangitis or cholecystitis.

4. History of cholecystectomy.

5. Considered unsuitable for participation in the study by their attending physician.

\section{Schedule of the study}

After obtaining informed consent, researchers will check the inclusion and exclusion criteria, participants' background and performance status. Within 28 days before surgery, blood test values such as complete blood count, aspartate aminotransferase alanine aminotransferase, $\gamma$-glutamyl transpeptidase, alkaline phosphatase, total bilirubin, albumin, blood urea nitrogen, creatinine, $\mathrm{C}$ reactive protein, serum sodium, serum potassium, carcinoembryonic antigen and CA19-9 antigen, alpha fetoprotein, Des-gamma carboxyprothrombin, indocyanine green test and radiological findings will be evaluated. After surgery, blood tests will be performed on postoperative days $0,1,3,5$ and 7 to detect adverse effect of the leak test; direct bilirubin from the drainage tube will be measured to detect the existence of bile leak.

\section{Intervention}

After completion of liver parenchymal dissection, the leak test is performed with CE-IOUSC. At first, a catheter is inserted from the cystic duct to the common bile duct. Under clamping of the lower common bile duct by 
hand, a mixed solution of indigo carmine as dye agent and 500-fold diluted Sonazoid as contrast agent for ultrasonography is injected. Sufficient intrahepatic biliary enhancement near the cut surface of the liver will be identified using ultrasonography in Sonazoid mode and then bile leak will be checked. If the intrahepatic biliary duct is not identified after three times of challenges, the test will be stopped and considered to be failure. Leak test will be performed by three experienced surgeons. For the decision of bile leak, the surgeon visually checks the existence of dye. To evaluate adverse event of biliary injection of Sonazoid, intraoperative and postoperative monitoring of vital signs and blood test results will be scheduled until discharge from the hospital.

\section{Outcomes}

\section{Primary endpoint}

The primary endpoint is the success of leak test after liver resection. When the intrahepatic bile ducts near cut surface of liver can be clearly visualised by ultrasonography after injecting Sonazoid mixed with dye agent, leak test will be considered to be successful because it means enough volume of mixed solution reaches to peripheral bile duct. If the intrahepatic bile ducts are not visualised, biliary injection will be repeated up to three times and success or failure will be judged. Information of injection volume, number of times, the presence of bile leakage after test and the way to repair bile leak is also recorded.

\section{Secondary endpoints}

Secondary endpoints are postoperative bile leak and all adverse events related to CE-IOUSC. Postoperative bile leak will be evaluated according to International Study Group of Liver Surgery (ISGLS) grading, reported in

Table 1 ISGLS Definition and Grading of bile leakage after hepatobiliary surgery

\section{Definition}

Bile leakage is defined as fluid with an increased bilirubin concentration in the abdominal drain or in the intraabdominal fluid on or after postoperative day 3 or as the need for radiological intervention because of biliary collections or relaparotomy resulting from bile peritonitis. Increased bilirubin concentration in the drain or intraabdominal fluid is defined as a bilirubin concentration at least three times greater than the serum bilirubin concentration measured at the same time.

\section{Grade}

$\begin{array}{ll}\text { A } & \begin{array}{l}\text { Bile leakage requiring no or little change in } \\ \text { patients' clinical management }\end{array} \\ \text { B } & \begin{array}{l}\text { Bile leakage requiring a change in patients } \\ \text { clinical management (eg, additional diagnostic } \\ \text { or interventional procedures) but manageable } \\ \text { without relaparotomy or a Grade A bile leakage } \\ \text { lasting for }>1 \text { week }\end{array} \\ \text { C } & \text { Bile leakage requiring relaparotomy }\end{array}$

ISGLS, International Study Group of Liver Surgery.
$2011^{20}$ (table 1), until discharge from the hospital. The occurrence and grade of bile leak will be analysed. For adverse events, we will investigate the presence of changes in vital signs during surgery and data of postoperative blood tests. Adverse events will be monitored by anaesthesiologist, surgeons and medical staff and evaluated using Clavien-Dindo classification. ${ }^{21}$ The study group will discuss each adverse event based on patient's background and surgical procedure as to distinguish CE-IOUSC related reaction from surgery-related reaction and principle investigator will determine.

\section{Sample size}

The purpose of primary analysis of this study is to estimate proportion of success of the leak test with CE-IOUSC during hepatectomy. When the expected proportion of success is $95 \%$, the two-sided 95\% CI width is calculated as 0.09 , and the required number of subjects is calculated to be 95 . By assuming five dropouts, the target sample size of this study has been set to 100 .

Since the number of liver resections per year in Kobe University Hospital is more than 100 , it is possible to accumulate the required number of subjects in 3 years of the registration period, even when considering cases in whom informed consent is not obtained.

\section{Data management}

A researcher will document in writing and submit the case report form (CRF) to the study committee before liver resection (screening test), during operation (intraoperative leak test) and after operation until discharge (postoperative bile leak and blood test). The research committee will prepare the dataset from the CRFs. After all participants have finished the study course, the data will be reviewed and will be corrected if needed. All analyses will be performed on the fixed data.

\section{Data analysis}

Analysis populations

The full analysis set (FAS) will consist of all subjects enrolled in this study, who undergo the leak test at least once and with efficacy data available, excluding those without baseline data or with significant protocol violations (eg, absence of informed consent, enrolment outside the contract period). The per protocol set (PPS) will consist of the subjects without significant protocol violations in the leak test with CE-IOUSC procedure, inclusion and exclusion criteria and concomitant therapies. The safety analysis set will consist of all subjects enrolled in this study and given at least one of the leak test.

The data analysis will be performed after data lock after completion of data collection. For all efficacy endpoints, the FAS will be used in the primary analysis, while the PPS will be used in a reference analysis. Safety will be analysed using the safety analysis set. All analysis will be performed with fixed data. All statistical analyses will be conducted using SPSS software (V.22.0, Chicago, Illinois, USA). 
Handling of cases

Inclusion of enrolled subjects for analyses will be judged by the consensus between the study representative and the responsible biostatistician. Handling of any problematic cases newly occurring in the study will also be determined by discussion between the study representative and the responsible biostatistician.

\section{Handling of data}

For data summarisation or analysis, in principle, the data will be handled as follows. When any doubt has arisen, the responsible biostatistician and the study representative will discuss to decide the data handling. Missing values will be imputed as necessary.

\section{Summarisation of subject baseline characteristics}

For the subject baseline characteristics data, the distribution and summary statistics will be calculated. For nominal variables, the categorical frequency and proportion will be presented. For continuous variables, the summary statistics (number of subjects, mean, SD, minimum, median and maximum) will be calculated.

\section{Efficacy analysis}

Point estimates and the 95\% CI of the proportion of success of the bile leak test and rate of bile leak after liver will be calculated.

\section{Safety analysis}

The safety endpoint of this study is the frequency of adverse events. A summary table will be prepared for the endpoint (table 2). For estimation of the proportion, the exact two-sided $95 \%$ CI for binomial distribution will be calculated by group.

\section{Data and safety monitoring}

Independent data and safety monitoring will be conducted. The following materials will be reviewed every 6 months: informed consent obtained and signed, participant retention, study implementation system, study safety and data and study progress.

\section{Patients and public involvement}

Neither patients nor the public were involved in the current study.

\section{Confidentiality}

All study-related information will be stored securely at the study site and identified by a coded number only to maintain participant confidentiality. All participant information will be stored in locked file cabinets in areas with limited access. All records that contain names or other personal identifiers will be stored separately from study records identified by code number. All local databases will be secured with password-protected access systems. Participants' study information will not be released outside of the study without the written permission of the participant.

\section{ETHICS AND DISSEMINATION}

This study will be conducted according to the declaration of Helsinki, 'Clinical Trials act' in Japan and Management Guidelines of Conflict of Interest in our institution. The protocol was approved by Institutional Review Boards of Kobe University Hospital on 20 May 2018 (No 290069). When revising the protocol, the principal investigator will discuss with the subinvestigator and obtain approval from Institutional Review Boards of Kobe University Hospital. Additionally, if there are significant changes,

\begin{tabular}{|c|c|c|c|c|c|c|c|c|}
\hline \multirow[b]{3}{*}{$\begin{array}{l}\text { TImepoint } \\
\text { (Day) }\end{array}$} & \multicolumn{8}{|l|}{ Study period } \\
\hline & \multirow{2}{*}{$\begin{array}{l}\begin{array}{l}\text { Recruitment/ } \\
\text { Screening }\end{array} \\
-28\end{array}$} & \multirow{2}{*}{$\begin{array}{l}\text { Allocation } \\
-14\end{array}$} & \multicolumn{6}{|c|}{ Postallocation } \\
\hline & & & $\begin{array}{l}0 \\
\text { (Operation) }\end{array}$ & 1 & 3 & 5 & 7 & At discharge \\
\hline Agreement & $\mathrm{x}$ & & & & & & & \\
\hline Patient background & $x$ & & & & & & & \\
\hline Performance status & $\mathrm{x}$ & & & & & & & \\
\hline Blood test & $\mathrm{x}$ & & $\mathrm{x}$ & $x$ & $x$ & $x$ & $x$ & $\mathrm{x}$ \\
\hline Vital sign & $\mathrm{x}$ & & $x$ & $\mathrm{x}$ & $\mathrm{x}$ & $\mathrm{x}$ & $x$ & $\mathrm{x}$ \\
\hline Allocation & & $\mathrm{x}$ & & & & & & \\
\hline Intervention & & & $x$ & & & & & \\
\hline \multicolumn{9}{|l|}{ Assessments } \\
\hline Success of leak test & & & $x$ & & & & & \\
\hline Adverse effects & & & $x$ & $x$ & $x$ & $x$ & $x$ & $\mathrm{x}$ \\
\hline Bile leak & & & $\mathrm{x}$ & $\mathrm{x}$ & $\mathrm{x}$ & $x$ & $\mathrm{x}$ & $\mathrm{x}$ \\
\hline Other complications & & & $\mathrm{x}$ & $\mathrm{x}$ & $\mathrm{x}$ & $\mathrm{x}$ & $x$ & $x$ \\
\hline
\end{tabular}


the investigator will explain those to the research participants and reobtain consent.

If a subject suffers health injury related to this study, the subinvestigator will perform appropriate action and treatment. For compensation of health injury, a clinical study liability insurance policy will be taken out to compensate for death or permanent disability (grade 1 or 2 permanent disability) resulting from this study. For other health injury, the tests, treatments and other necessary procedures will be performed within the range of medical services covered by the subject's health insurance.

The results of this study will be disseminated through academic conferences and peer-reviewed journals. All authors will review and approve the paper before publication. If there will be several papers related to this study, the investigator will appoint each author.

\section{DISCUSSION}

Bile leak remains one of the major unsolved morbidities of liver resection. The leak test has been reported to be effective for detecting leak point intraoperatively and preventing postoperative bile leak. ${ }^{12-15}$ However, in the conventional leak test, it is sometimes difficult to identify injecting test reagent into the intrahepatic bile duct when confluence of the cystic duct is at a lower level of the distal common bile duct. Too much volume of injection has a possibility to make a secondary injury of bile duct and too little volume of injection results in lower detection rate of bile leak. To know whether test reagent is injected with adequate volume and pressure, CE-IOUSC will be helpful. We have reported the usefulness of CE-IOUSC to visualise and detect the intrahepatic bile duct with a very high success rate of $96 \% .{ }^{17}$ Using this technique, visualisation of the test reagent injected into the hepatic duct is possible. Because this procedure enables the real time biliary navigation, we can stop injection just after the microbubble reaching to cut surface to prevent secondary bile duct injury by too much pressure. As a procedure for visualisation of the test reagent, air leak test was reported to be useful to confirm the injected air by ultrasonography. ${ }^{14}$ However, injected air is sometimes seen like an artefact and can be unclear on ultrasonography. On the other hand, our procedure enables much clearer visualisation of the non-dilated intrahepatic bile duct, ${ }^{17}$ which means more precise and strict leak test for checking minor/major bile leak points, potentially leading to the consequent reduction in postoperative bile leak.

There are two types of bile leak. One is leakage from intrahepatic bile duct connected to common bile duct (dependent site) and the other is from peripheral bile duct unconnected to common bile duct (non-dependent site). Generally, leak test is a procedure to detect leak from dependent site, not non-dependent site. Our procedure using CE-IOUSC is also same as conventional test.

Because this study is a single-arm study with off-label use of the contrast-enhancing agent, the safety of biliary injection of mixed solution of Sonazoid and indigo carmine needs to be evaluated. Vital sign and blood data will be monitored, but it may be difficult to clearly distinguish adverse events by CE-IOUSC from those by surgery. Therefore, adverse event will to be evaluated by study group and determined by principle investigator according to patient background and surgical procedure in each case.

The findings obtained through this study will help establish the procedure of CE-IOUSC in hepatobiliary surgery, which may assist surgeons in adequately performing the leak test, detecting intraoperative biliary leak strictly and reducing postoperative bile leak.

The current study will provide fundamental data for the optimisation of CE-IOUSC, serving as a prerequisite in planning the next confirmative study, to establish clinical utility of CE-IOUSC in the liver surgery.

Contributors All the authors contributed to study design and writing and revising the protocol. TF (chief investigator) and MT (principle investigator) are responsible for the original concept and study design. MT drafted the initial protocol. TF, MK and MT have a role in liver resection and leak test as a surgeon. KK, SK, MA and HG have a role of patient recruitment and perioperative care. DT, HM and HT will be responsible for data statistics, data management and data monitoring, respectively. All authors have read and approved the final study protocol.

Funding The authors have not declared a specific grant for this research from any funding agency in the public, commercial or not-for-profit sectors.

Competing interests None declared.

Patient consent for publication Not required.

Ethics approval The Institutional Review Board of Kobe University.

Provenance and peer review Not commissioned; externally peer reviewed.

Open access This is an open access article distributed in accordance with the Creative Commons Attribution Non Commercial (CC BY-NC 4.0) license, which permits others to distribute, remix, adapt, build upon this work non-commercially, and license their derivative works on different terms, provided the original work is properly cited, appropriate credit is given, any changes made indicated, and the use is non-commercial. See: http://creativecommons.org/licenses/by-nc/4.0/.

\section{REFERENCES}

1. Asiyanbola B, Chang D, Gleisner AL, et al. Operative mortality after hepatic resection: are literature-based rates broadly applicable? J Gastrointest Surg 2008;12:842-51.

2. Belghiti J, Hiramatsu K, Benoist S, et al. Seven hundred forty-seven hepatectomies in the 1990s: an update to evaluate the actual risk of liver resection. J Am Coll Surg 2000;191:38-46.

3. Hyder O, Pulitano C, Firoozmand A, et al. A risk model to predict 90 -day mortality among patients undergoing hepatic resection. J Am Coll Surg 2013;216:1049-56.

4. Imamura H, Seyama $\mathrm{Y}$, Kokudo $\mathrm{N}$, et al. One thousand fiftysix hepatectomies without mortality in 8 years. Arch Surg 2003;138:1198-206. discussion 206.

5. Jarnagin WR, Gonen M, Fong Y, et al. Improvement in perioperative outcome after hepatic resection: analysis of 1,803 consecutive cases over the past decade. Ann Surg 2002;236:397-406. discussion -7.

6. Ferrero A, Russolillo N, Viganò L, et al. Safety of conservative management of bile leakage after hepatectomy with biliary reconstruction. J Gastrointest Surg 2008;12:2204-11.

7. Guillaud A, Pery C, Campillo B, et al. Incidence and predictive factors of clinically relevant bile leakage in the modern era of liver resections. HPB 2013;15:224-9.

8. Tanaka S, Hirohashi K, Tanaka H, et al. Incidence and management of bile leakage after hepatic resection for malignant hepatic tumors. $J$ Am Coll Surg 2002;195:484-9.

9. Zimmitti G, Roses RE, Andreou A, et al. Greater complexity of liver surgery is not associated with an increased incidence of liver-related complications except for bile leak: an experience with 2,628 consecutive resections. J Gastrointest Surg 2013;17:57-65. discussion 5 . 
10. Martin AN, Narayanan S, Turrentine FE, et al. Clinical factors and postoperative impact of bile leak after liver resection. $J$ Gastrointest Surg 2018;22:661-7.

11. Yun SU, Cheon YK, Shim CS, et al. The outcome of endoscopic management of bile leakage after hepatobiliary surgery. Korean $J$ Intern Med 2017;32:79-84.

12. Liu Z, Jin H, Li Y, et al. Randomized controlled trial of the intraoperative bile leakage test in preventing bile leakage after hepatic resection. Dig Surg 2012;29:510-5.

13. Wang $\mathrm{HQ}$, Yang J, Yang JY, et al. Bile leakage test in liver resection: a systematic review and meta-analysis. World $J$ Gastroenterol 2013;19:8420-6.

14. Zimmitti G, Vauthey JN, Shindoh J, et al. Systematic use of an intraoperative air leak test at the time of major liver resection reduces the rate of postoperative biliary complications. J Am Coll Surg 2013;217:1028-37.

15. Tran Cao HS, Phuoc V, Ismael H, et al. Rate of Organ Space Infection Is Reduced with the Use of an Air Leak Test During Major Hepatectomies. J Gastrointest Surg 2017;21:85-93.
16. Linke R, Ulrich F, Bechstein WO, et al. The White-test helps to reduce biliary leakage in liver resection: a systematic review and metaanalysis. Ann Hepatol 2015;14:161-7.

17. Urade T, Fukumoto T, Tanaka M, et al. Contrast-enhanced intraoperative ultrasonic cholangiography for real-time biliary navigation in hepatobiliary surgery. J Am Coll Surg 2014;218:e43-50.

18. Urade T, Fukumoto T, Kido M, et al. Contrast-enhanced intraoperative ultrasonic cholangiography in living donor hepatectomy. Liver Transp/ 2016;22:1437-42.

19. Chan AW, Tetzlaff JM, Altman DG, et al. SPIRIT 2013 statement: defining standard protocol items for clinical trials. Ann Intern Med 2013;158:200-7.

20. Koch M, Garden OJ, Padbury R, et al. Bile leakage after hepatobiliary and pancreatic surgery: a definition and grading of severity by the International Study Group of Liver Surgery. Surgery 2011;149:680-8.

21. Dindo D, Demartines N, Clavien P-A. Classification of Surgical Complications. Ann Surg 2004;240:205-13. 